\title{
Variáveis Psicossociais e Desempenho em Leitura de Crianças de Baixo Nível Socioeconômico
}

\author{
Luciane da Rosa Piccolo \\ Olga Garcia Falceto \\ Universidade Federal do Rio Grande do Sul \\ Carmen Luisa Fernandes \\ Grupo Hospitalar Conceição \\ Daniela Centenaro Levandowski \\ Universidade Federal de Ciências da Saúde de Porto Alegre \\ Rodrigo Grassi-Oliveira \\ Pontificia Universidade Católica do Rio Grande do Sul \\ Jerusa Fumagalli Salles \\ Universidade Federal do Rio Grande do Sul
}

\begin{abstract}
RESUMO - Este estudo longitudinal investigou a contribuição de fatores psicossociais para o desempenho em leitura de crianças. Participaram 59 mães e seus filhos aos 4 meses, 2, 5-6 e 9-11 anos. Correlacionou-se o desempenho em leitura das crianças a variáveis psicossociais. Encontrou-se correlações negativas entre morbidade psiquiátrica materna na primeira infância e leitura de palavras irregulares e entre o número de familiares que residiam com a criança aos 2 e 5-6 anos e o desempenho na leitura de palavras. A renda familiar aos 2 anos correlacionou-se positivamente com compreensão textual. Entretanto, apenas o número de familiares que residiam com a criança foi preditor do desempenho em leitura de palavras. Conclui-se que o desempenho em leitura relaciona-se a fatores psicossociais.
\end{abstract}

Palavras-chave: fatores psicossociais, desempenho em leitura, compreensão de leitura, família.

\section{Psychosocial Variables and Reading Performance of Children with Low Socioeconomic Status}

\begin{abstract}
This longitudinal study investigated the impact of psychosocial factors on reading performance of children with low socioeconomic status. Fifty nine mothers and their children at 4 months, 2, 5-6 and 9-11 years of age participated. The children's reading performance was correlated with psychosocial variables. Negative correlations were found between maternal psychiatric morbidity in early childhood and reading of irregular words, and between the number of family members living with the child at 2 and 5-6 years and word reading performance. Family income at 2 years correlated positively with reading comprehension. However, only the number of family members who lived with the child was a predictor of reading performance. The results indicate that reading performance is related to psychosocial factors.
\end{abstract}

Keywords: psychosocial factors, reading performance, reading comprehension, family.

Embora inicialmente os estudos sobre desempenho em leitura tenham dado atenção a um ou outro aspecto, em geral de cunho cognitivo, relacionado a essa habilidade, mais recentemente tem se percebido uma perspectiva mais ampla e integradora no entendimento desse processo. Nesse sentido, Aaron e colaboradores (Aaron, Joshi, Gooden, \& Bentum, 2008; Joshi \& Aaron, 2000) propuseram um modelo de leitura de múltiplos componentes, segundo o qual o desenvolvimento e o desempenho de tal habilidade sofreriam influência de fatores cognitivos, ambientais (ecológicos) e psicológicos. Segundo os autores, o domínio cognitivo envolveria reconhecimento de palavras e compreensão e outras habilidades cognitivas relacionadas à leitura (como a memória, por exemplo). O domínio psicológico abrangeria aspectos como motivação e interesse, locus de controle,

1 Apoio: Capes, CNPq 574819/2008-8.

2 Endereço para correspondência: Rua Ramiro Barcellos, 2600, sala 114. Porto Alegre, RS.CEP90035-003.E-mail: luciane-piccolo@hotmail.com. desamparo aprendido, estilos de aprendizagem, expectativa dos professores e diferenças de gênero. Por fim, o domínio ecológico incluiria aspectos do ambiente doméstico e da cultura, o envolvimento dos pais, o ambiente de sala de aula e o uso de dialetos, por exemplo.

Da mesma forma, Fletcher, Lyons e Barnes (2007) sugerem um modelo geral para o entendimento dos transtornos de aprendizagem, incluindo a leitura. Nesse modelo, são considerados três níveis de análise: 1) habilidades acadêmicas específicas (reconhecimento de palavras, compreensão e fluência na leitura, etc); 2) características da criança, incluindo os processos cognitivos, motivação, habilidades sociais ou problemas comportamentais; e 3) fatores neurobiológicos e ambientais (situações sociais e econômicas). Dessas abordagens, destaca-se a importância de considerar diversos fatores, como os psicossociais, para a compreensão do desempenho em leitura. A seguir, serão exploradas as relações entre os fatores psicossociais e tal habilidade. 


\section{Relação entre Fatores Psicossociais e Desempenho em Leitura}

A importância dos fatores psicológicos e do ambiente familiar para o aprendizado é reconhecida pelos educadores e tem sido empiricamente documentada (Aaron et al., 2008). De fato, a literatura tem apontado alguns desses fatores, que parecem influenciar o aprendizado escolar geral, tais como: nível socioeconômico (Andrada, Benetti, Carvalho, \& Rezena, 2008; Noble, Wolmetz, Ochs, Farah, $\&$ McCandliss, 2006; Peeters, Verhoeven, Moor, Balkom, \& Leeuwe, 2009; Skibbe, Justice, Zucker, \& McGinty, 2008), oferta de recursos físicos (disponibilidade de passeios, brinquedos e jogos adequados para a idade da criança) (Marturano, 2000, 2006; Marturano, Alves, \& Santa Maria, 1996), exposição à leitura no ambiente doméstico (Duursma, Pan, \& Raikes, 2008; Haney \& Hill, 2004; Kuo, Franke, Regalado, \& Halfon, 2004; Sénéchal \& LeFreve, 2002), organização e recursos do lar (condições físicas satisfatórias da moradia e ambiente tranquilo e acolhedor) (D'Avila-Bacarji, Marturano, \& Elias, 2005; Oliveira et al, 2005), número de pessoas da família (D'Avila-Bacarji, Marturano \& Elias, 2005; Ferreira \& Marturano, 2002; Okano, Loureiro, Linhares, \& Marturano., 2004), número de filhos (Ferreira \& Marturano, 2002; Marturano, 2006; Okano, Loureiro, Linhares \& Marturano, 2004), envolvimento dos pais com a vida escolar da criança (Marturano et al. 1996; Okano et al. 2004), escolaridade e idade dos pais (D'Avila-Bacarji, et al. 2005; Kuo et al., 2004; Skibbe et al., 2008) e depressão materna (Snowling, Muter, \& Carroll, 2007; Stanley, Murray, \& Stein, 2004). Como visto, a família pode oferecer às crianças apoio para o desempenho escolar, por meio do envolvimento dos pais na vida acadêmica dos filhos, da organização das rotinas no lar e de oportunidades de interação com os pais (D'Avila-Bacarji et al. 2005; Marturano, 2000, 2006; Marturano et al. 1996), por exemplo. Da mesma forma, pode oferecer suporte para o seu desenvolvimento cognitivo, através de atividades recreativas e educativas, da oferta de brinquedos, livros e outros materiais. Além disso, a família pode oferecer apoio emocional, a partir de um ambiente doméstico acolhedor (D'Avila-Bacarji et al. 2005). Mais do que isso, o bom relacionamento conjugal e entre pais e filhos tem uma repercussão positiva sobre a motivação para a aprendizagem (Carlton \& Winsler, 1999; Marturano \& Ferreira, 2004).

Dentre esses aspectos familiares, a exposição à leitura no ambiente doméstico mostra-se bastante relevante para o desempenho escolar da criança (Duursma et al., 2008; Haney \& Hill, 2004; Kuo et al., 2004) e, mais especificamente, para o reconhecimento de palavras (Peeters et al., 2009) e a consciência fonológica, um dos melhores preditores da leitura proficiente (Foy \& Mann, 2003; Kim, 2009). As mães com maior nível de educação parecem ser mais propensas a ler com frequência para seus filhos do que mães com níveis mais baixos de escolaridade (Kuo et al., 2004; Skibbe et al., 2008). Por outro lado, quando a família encontra dificuldades para cumprir adequadamente suas tarefas básicas de socialização e de amparo aos seus membros, criam-se situações de vulnerabilidade. Todos aqueles elementos que potencializam situações de risco podem remeter à vulnerabilidade
(Hutz, Koller, \& Bandeira, 1996). A pobreza, por exemplo, é considerada um tipo de ameaça constante, que aumenta a vulnerabilidade da criança, pois pode causar subnutrição, privação social e desvantagem educacional (Zimmerman \& Arunkumar, 1994), limitando as oportunidades de desenvolvimento (Ceconello \& Koller, 2000). Nessa situação, as crianças podem apresentar dificuldades de atenção, o que limita a aquisição de informações e a capacidade de aprender (Smith \& Strick, 2001).

Apesar da extensa produção científica sobre a contribuição do ambiente familiar para o desempenho escolar, os fatores psicossociais que influenciam especificamente o desenvolvimento e estão subjacentes às dificuldades de leitura encontram-se bem menos definidos e explorados na literatura. Sabe-se que o ambiente é importante, porque fornece estímulos e possibilita experiências fundamentais para o desenvolvimento da linguagem (incluindo a linguagem escrita) como meio principal de interação social (Kim, 2009; Oliveira et al., 2005; Peeters et al., 2009). Nesse sentido, os poucos estudos que investigaram especificamente a relação do ambiente familiar com a leitura encontraram, basicamente, os mesmos resultados apontados pelas investigações sobre o desempenho acadêmico geral. Em sua maioria, referem-se, por exemplo, à exposição à leitura (Duursma et al., 2008; Foy \& Mann, 2003; Haney \& Hill, 2004; Sénéchal \& LeFreve, 2002), à alta escolaridade dos pais (Kuo et al., 2004) e à melhor condição socioeconômica (Foy \& Mann, 2003) como fatores de proteção para o desenvolvimento e o desempenho da leitura.

Tendo em vista a necessidade de melhor explorar quais aspectos do ambiente são importantes para o desempenho em leitura, o presente estudo examinou a relação entre fatores psicossociais (socioeconômicos e familiares), investigados em três momentos do desenvolvimento (4 meses, 2 anos, $5 / 6$ anos de idade) e o desempenho em leitura, avaliado aos 9-11 anos, de crianças de uma coorte populacional de baixo nível socioeconômico.

\section{Método}

\section{Participantes}

A amostra desse estudo foi constituída de 59 crianças, sendo 29 meninos $(49,2 \%)$ e 30 meninas $(50,8 \%)$, estudantes de $2^{\mathrm{a}}$ a $5^{\mathrm{a}}$ séries do ensino fundamental de escolas públicas, e suas mães, todos provenientes de um estudo de coorte (Falceto, 2002; Falceto et al., 2007). Na quarta etapa do estudo, realizada em 2009, na qual a leitura das crianças foi avaliada, as mesmas tinham idade média de 10,4 anos (mínimo 9,7 e máximo 11,08 anos, $D P=0,44$ ). As famílias eram de nível socioeconômico baixo, com renda familiar média de quatro salários mínimos $(M=3,95, D P=2,5)$ aproximadamente. Na primeira fase de coleta de dados (quatro meses do bebê), as mães das crianças tinham entre 14 e 42 anos $(M=24,12$ e $D P=6,29)$, sua escolaridade variava entre um e 14 anos de estudo formal $(M=6,84 \mathrm{e}$ $D P=2,61)$ e sua renda média era de 1,15 salários mínimos $(D P=3,61)$. 


\section{Instrumentos e Procedimentos}

Através dos registros dos nascimentos dos hospitais da cidade de Porto Alegre no período de dezembro de 1998 a dezembro de 1999, foram identificadas as famílias dos bebês da área de abrangência do estudo. Logo após o bebê completar quatro meses, um estudante de Medicina fazia uma visita domiciliar para obter dados de identificação e o consentimento das famílias para a participação na pesquisa. Nas primeiras três etapas do estudo de coorte (aos 4 meses, 2 e 5/6 anos da criança) foram realizadas entrevistas sociodemográficas com os pais das crianças e a aplicação de instrumentos para investigar diferentes informações individuais, do casal e da família. Mais detalhes sobre os instrumentos empregados nessas etapas podem ser encontrados em Falceto et al. (2007). No presente estudo foram utilizados apenas dados das mães das crianças referentes a essas etapas. No ano de 2009, essas famílias foram recontatadas para que se procedesse à avaliação das crianças. Tais avaliações foram realizadas nas escolas das crianças, mediante contato prévio e autorização da Direção.

A seguir, serão descritas as variáveis aqui consideradas em cada etapa do estudo longitudinal. Cabe salientar que o projeto do qual esse estudo deriva foi aprovado pelo Comitê de Ética do Hospital de Clínicas de Porto Alegre.

$\mathrm{Na}$ primeira etapa (4 meses da criança), foram consideradas as variáveis idade materna (em anos), escolaridade materna (em anos), renda média materna e familiar (em salários mínimos), número de familiares que residiam com a criança (número de avós, primos, irmãos, tios e os pais das crianças estudadas, que habitavam a mesma moradia), número de horas que a mãe passa com a criança. Dados das entrevistas serviram para a descrição da amostra (nível de pobreza e classe social). Ainda, foi considerado o escore materno no Questionário de Auto-Relato (Self-Reporting Questionnaire - SRQ-20) (WHO, 1994), que contém 20 questões para a avaliação de transtornos não-psicóticos, com duas possibilidades de resposta ( $\operatorname{sim} /$ não). O instrumento teve como objetivo detectar sintomas emocionais e físicos associados a quadros psiquiátricos. Na padronização brasileira, foi verificada consistência interna de 0,80 (Mari \& Williams, 1986).

Na segunda etapa (2 anos da criança), as variáveis consideradas foram as mesmas descritas na primeira etapa, com exceção da idade e escolaridade da mãe. Por outro lado, foram analisados também os escores maternos no Inventário de Depressão de Beck (Beck Depression Inventory - BDI) (Beck, Ward, Mendelson, Mock, \& Erbaugh, 1961; Beck \& Steer, 1993; Cunha, 2001). Constituído de 21 itens, com quatro possibilidades de resposta cada um, variando de zero a três, esse inventário é utilizado para avaliar a intensidade de sintomas depressivos. A soma dos escores dos itens individuais fornece um escore total, que se classifica em níveis de intensidade. As normas da versão brasileira classificam a intensidade dos sintomas em mínima (0-11), leve (12-19), moderada (20-35) (Cunha, 2001).

Foram ainda utilizados indicadores de risco da criança (Farjado et al., 2004) e o Teste de DENVER II (Frankenburg, Dodds, Archer, Shapiro, \& Bresnick, 1992). Este último foi utilizado para avaliar suspeita de transtorno de desenvolvi- mento nas crianças estudadas, dividindo-se aquelas com e sem suspeita. O teste consiste em 125 itens, divididos em quatro grupos: a) pessoal/social; b) motricidade fina; c) linguagem e d) motricidade ampla. Esses itens foram registrados através de observação direta da criança e, para alguns deles, solicitou-se que a mãe informasse se o filho realizava ou não determinada tarefa. O teste Denver II apresenta bons índices de validade e confiabilidade $(0,99$ interobservador e 0,9 em teste-reteste), por isso, é amplamente utilizado (Santos, Araújo, \& Porto, 2008). Já o instrumento Indicadores de Risco da Criança (Farjado et al., 2004) foi utilizado para avaliar características capazes de influenciar no crescimento e no desenvolvimento infantil. As variáveis utilizadas foram aquelas consideradas pela Secretaria Municipal de Saúde de Porto Alegre e pelo Serviço de Saúde Comunitária do Grupo Hospitalar Conceição para avaliar o risco em bebês: peso ao nascer, duração da gestação, aleitamento materno, gestação gemelar, hospitalização, malformação congênita, idade materna, escolaridade materna, óbito de irmão, número de filhos residindo na mesma casa, número de consultas de pré-natal, renda per capita, problemas familiares e socioeconômicos, vacinas, avaliação clinica de desenvolvimento e indicadores de desenvolvimento e crescimento (altura/idade, peso/altura, peso/idade). Considera-se "alto risco" quando o total de pontos somados nos 19 indicadores for maior ou igual a seis.

$\mathrm{Na}$ terceira etapa (5-6 anos da criança, 2005): foram consideradas as mesmas variáveis das etapas anteriores. Entretanto, foi utilizado o BDI-PC (Beck Depression Inventory for Primary Care) (Beck, Guth, Steer, \& Ball, 1997) e o checklist do DSM-IV-TR na avaliação da psicopatologia materna. O BDI-PC, escala de rastreio que contém sete itens do BDI, foi usado para investigar indícios de depressão nas mães das crianças. Nos estudos de normatização do teste com adolescentes, o instrumento apresentou alta consistência interna (alpha de Cronbach $=0,88$ ) (Winter, Steer, Jones-Hicks, \& Beck, 1999). Por sua vez, o checklist do DSM-IV-TR (American Psychiatric Association, 2002) foi utilizado pelos terapeutas que entrevistaram as famílias para verificar a existência de algum tipo de transtorno psiquiátrico nas mães das crianças.

$\mathrm{Na}$ quarta etapa (9-11 anos da criança, 2009): foram considerados os escores das crianças para leitura de palavras isoladas (Salles \& Parente, 2002a; 2002b), compreensão de leitura textual (Salles \& Parente, 2004) e inteligência - subtestes vocabulário e raciocínio matricial da Wechsler Abbreviated Scale of Intelligence - WASI (The Psychological Corporation, 1999; Yates et al., 2006). Os escores neste teste foram utilizados como variável de controle nas análises. A WASI é um instrumento administrado individualmente, para idades de seis a 89 anos, composta por quatro subtestes Vocabulário, Cubos, Semelhanças e Raciocínio Matricial -, que avaliam aspectos cognitivos, como conhecimento verbal, processamento de informação visual, raciocínio espacial e não verbal, inteligência fluída e cristalizada. O processo de adaptação, validação e normatização da WASI para a população brasileira está em desenvolvimento (Yates et al., 2006). Neste estudo, foi utilizada a versão reduzida desse instrumento (subtestes vocabulário e raciocínio matricial). A avaliação de leitura de palavras isoladas (Salles \& Parente, 2002a, 2002b, 2007) foi utilizada para verificar 
a capacidade de leitura de palavras reais e pseudopalavras das crianças. A avaliação da compreensão de leitura textual (Salles, 2005, Salles \& Parente, 2002a, 2004, 2009) baseia-se nas respostas das crianças às questões sobre uma história lida. Para este estudo, foi considerada a quantidade de acertos obtidos nas dez questões. As aplicações dos instrumentos foram realizadas de forma randomizada, ou seja, metade da amostra respondeu primeiramente a WASI e, posteriormente, as tarefas de leitura, enquanto que a outra metade respondeu as tarefas de leitura primeiramente e depois a WASI. Para este estudo, foi considerada a quantidade de acertos obtidos nas dez questões.

\section{Resultados}

Em relação ao desenvolvimento das crianças, observou-se que, na segunda etapa da pesquisa, $70,7 \%$ foram consideradas como sendo de "alto risco" pela medida "Indicadores de Risco para o Desenvolvimento Infantil". No caso, escores acima de seis apontam que a criança está em "alto risco" (nessa amostra: $M=8,93 ; D P=6,5$ ). Também foi observado que $22 \%(n=13)$ delas foram consideradas excessivamente agitadas por suas mães aos 5-6 anos de idade, conforme respostas às entrevistas. Nesse mesmo período, 59,3\% $(n=$ 32) delas apresentaram suspeita de transtorno de desenvolvimento, a partir da avaliação realizada pelo teste DENVER
II (Frankenburg et al., 1992). Por sua vez, 34\% $(n=20)$ das mães apresentaram problemas psiquiátricos, sendo que $17 \%(n=10)$ apresentaram sintomas de episódio depressivo maior, segundo impressão diagnóstica dos entrevistadores a partir da aplicação do Checklist do DSM-IV-TR (American Psychiatric Association, 2002). A Tabela 1 apresenta a análise descritiva dos fatores psicossociais (socioeconômicos, educacionais e de saúde da mãe e familiares) nas três etapas da pesquisa e a Tabela 2 apresenta o desempenho geral das crianças nas tarefas de leitura de palavras, compreensão textual e na avaliação cognitiva WASI. Não foram constatadas diferenças significativas entre as médias de desempenho nas tarefas de leitura e da WASI em relação à ordem de aplicação dos instrumentos (teste $t$ de Student). Por fim, a Tabela 3 mostra apenas as correlações (teste de Pearson) significativas ( $p$ $<0,05$ ou $p<0,01$ ) existentes entre os fatores psicossociais (socioeconômicos e familiares) e o desempenho em leitura das crianças.

Dos fatores familiares considerados, foi observada correlação negativa entre o escore da mãe no SRQ-20 (primeira etapa) e o total de acertos na leitura de palavras reais irregulares $(r=-0,278, p<0,05)$. Tal resultado aponta que, quanto mais problemas emocionais a mãe apresentou, menor foi o desempenho da criança nessa tarefa. Além disso, uma correlação significativa negativa foi encontrada entre o número de familiares que residiam com a criança (segunda etapa) e o escore total de leitura de palavras $(r=$

Tabela 1. Análise descritiva dos fatores psicossociais, por etapa de coleta de dados.

\begin{tabular}{|c|c|c|}
\hline Fatores psicossociais & Média & Desvio Padrão \\
\hline \multicolumn{3}{|l|}{ Primeira etapa - 1998 (4 meses da criança) } \\
\hline Idade da mãe & 23,95 & 6,46 \\
\hline Escolaridade da mãe (em anos) & 6,84 & 2,55 \\
\hline Número de horas que a mãe passa com a criança & 21,69 & 4,73 \\
\hline Número de familiares que moram com a criança & 4,93 & 1,99 \\
\hline Renda média da família (em salários mínimos) & 3,92 & 2,52 \\
\hline Renda média da mãe (em salários mínimos) & 0,703 & 1,19 \\
\hline SRQ - 20 da mãe & 5,50 & 3,93 \\
\hline \multicolumn{3}{|l|}{ Segunda etapa - 2001 ( 2 anos da criança) } \\
\hline Renda média da família (em salários mínimos) & 3,77 & 2,36 \\
\hline Renda média da mãe (em salários mínimos) & 0,59 & 0,81 \\
\hline SRQ - 20 da mãe & 4,38 & 3,23 \\
\hline Número de familiares que moram com a criança & 4,65 & 1,59 \\
\hline BDI da mãe & 7,37 & 8,10 \\
\hline \multicolumn{3}{|l|}{ Terceira etapa - 2005 (5 anos da criança) } \\
\hline Número total de familiares que moram com a criança & 4,94 & 1,84 \\
\hline Renda média da mãe (em salários mínimos) & 0,84 & 0,12 \\
\hline Renda média da família (em salários mínimos) & 2,58 & 0,23 \\
\hline Número de horas que a mãe passa com a criança & 15,88 & 8,79 \\
\hline SRQ - 20 da mãe & 5,29 & 3,84 \\
\hline BDI da mãe & 2,78 & 3,85 \\
\hline
\end{tabular}

Legenda: SRQ - 20: Questionário de auto-relato - Self Reporting Questionary; BDI: Inventário de Depressão Beck - Beck Depression Inventory. 
Tabela 2. Desempenho nas tarefas de leitura de palavras/pseudopalavras, compreensão de leitura textual e avaliação cognitiva (WASI) - escores e percentual de acertos.

\begin{tabular}{lcc}
\hline Tarefas de leitura & Média & $\begin{array}{c}\text { Desvio } \\
\text { Padrão }\end{array}$ \\
\hline $\begin{array}{l}\text { Leitura de palavras } \\
(\text { Máximo }=60)\end{array}$ & 53,25 & 6,56 \\
Palavras reais & $(88,76 \%)$ & \\
(Máximo $=40)$ & 36,47 & 4,85 \\
Palavras reais regulares & $(91,19 \%)$ & \\
(Máximo $=20)$ & 18,71 & 2,31 \\
Palavras reais irregulares & $(93,56 \%)$ & \\
(Máximo $=20)$ & 17,44 & 2,82 \\
Pseudopalavras & $(87,20 \%)$ & \\
(Máximo $=20)$ & 17,10 & 2,43 \\
Compreensão de leitura textual & $(85,51 \%)$ & \\
(Máximo $=10)$ & 8 & 1,56 \\
$\begin{array}{l}\text { Vocabulário }- \text { WASI } \\
\text { (Máximo }=84 \text { pontos) }\end{array}$ & $(80 \%)$ & \\
$\begin{array}{l}\text { Raciocínio Matricial - WASI } \\
\text { (Máximo }=35)\end{array}$ & 22,05 & 7,60 \\
\hline
\end{tabular}

$-0,419, p<0,01)$, leitura de palavras reais $(r=-0,446, p$ $<0,01)$ e reais regulares $(r=-0,471, p<0,01)$. Da mesma forma, o número de familiares que residiam com a criança na terceira etapa correlacionou-se negativamente ao escore total de leitura de palavras $(r=-0,350, p<0,05)$, palavras reais $(r=-0,355, p<0,01)$ e palavras reais regulares $(r$ $=-0,367, p<0,01)$. Desse modo, percebe-se que, quanto mais numerosa a família, menor o escore em leitura das crianças estudadas.

Quanto aos fatores socioeconômicos, foi possível observar também que a renda familiar aos 2 anos da criança correlacionou-se significativa e positivamente com o escore em compreensão de leitura textual $(r=0,385, p<0,01)$. Quanto maior o status socioeconômico da família, mais alto o desempenho em compreensão de leitura das crianças. As demais variáveis psicossociais não apresentaram correlações significativas com o desempenho nas tarefas de leitura de palavras e de compreensão de texto.
Ainda, foi feita uma análise de regressão linear múltipla (método stepwise), na qual apenas os fatores psicossociais que apresentaram correlação significativa com as medidas de leitura entraram como preditores do desempenho em leitura de palavras isoladas e, depois, com o desempenho em compreensão de leitura, controlando-se o escore nas tarefas da WASI. Pretendia-se compreender quais fatores psicossociais contribuem para o desempenho em leitura, para, então, construir-se um modelo final. Foram analisados os seguintes modelos explicativos: 1) desempenho da mãe no SRQ-20 (primeira etapa) como preditor do desempenho de leitura de palavras reais irregulares; 2) número de familiares que residiam com a criança (segunda e terceira etapas) como preditor do desempenho no total de palavras, 3) palavras reais e 4) reais regulares e 5) renda média familiar (segunda etapa) como preditora do desempenho em compreensão textual. Todos os modelos foram testados com e sem o controle da medida de inteligência (subtestes da WASI).

$\mathrm{O}$ número de familiares que residiam com a criança (segunda etapa) mostrou-se preditor da leitura de palavras total $\left[R=0,42, R^{2}\right.$ ajustado $=0,16 ; F(1,46)=9,78, p<$ $0,05]$, palavras reais $\left[R=0,45, R^{2}\right.$ ajustado $=0,18 ; F(1,46)$ $=11,45, p<0,05]$ e palavras regulares $\left[R=0,47, R^{2}\right.$ ajustado $=0,20 ; F(1,46)=13,1, p<0,05]$. O número de familiares que residiam com a criança na terceira etapa foi preditor do desempenho em leitura de palavras total $\left[R=0,35, R^{2}\right.$ ajustado $=0,10 ; F(1,50)=6,7, p<0,05]$, palavras reais $[R$ $=0,35, R^{2}$ ajustado $\left.=0,11 ; F(1,50)=7,2, p<0,05\right]$ e reais regulares $\left[R=0,37, R^{2}\right.$ ajustado $=0,12 ; F(1,50)=7,8, p<$ $0,05]$. O desempenho da mãe no SRQ-20 (primeira etapa) não foi um preditor de leitura de palavras irregulares, independentemente do controle da medida de inteligência. Da mesma forma, a renda familiar (segunda etapa) não foi um preditor da compreensão de leitura.

Numa análise de correlação (Pearson) a posteriori observou-se que alguns dos fatores psicossociais também apresentaram relações significativas entre si. Houve correlações significativas negativas entre o número de familiares que residiam na casa com a criança e: 1$)$ BDI na primeira $(r$ $=0,43 ; p<0,01)$ e segunda etapas $(r=0,56 ; p<0,01) ; 2)$ número de filhos; 3$)$ SRQ- 20 na segunda etapa $(r=0,30$; $p<0,01)$; 4) quantidade de horas que a mãe passava com a criança; 5) renda familiar $(r=-0,37 ; p<0,01) ; 6)$ renda materna e 7) escolaridade da mãe. Além disso, houve corre-

Tabela 3. Matriz de Correlação (Pearson) entre fatores psicossociais e desempenho em leitura.

\begin{tabular}{|c|c|c|c|c|c|}
\hline Variáveis Psicossociais & $\begin{array}{l}\text { Leitura de } \\
\text { palavras }\end{array}$ & $\begin{array}{l}\text { Palavras } \\
\text { reais }\end{array}$ & $\begin{array}{l}\text { Palavras reais } \\
\text { regulares }\end{array}$ & $\begin{array}{l}\text { Palavras reais } \\
\text { irregulares }\end{array}$ & $\begin{array}{l}\text { Compreensão } \\
\text { textual }\end{array}$ \\
\hline SRQ - 20 da mãe (1ª etapa - 1998) & $-0,24$ & $-0,21$ & $-0,23$ & $-0,28^{*}$ & $-0,09$ \\
\hline $\begin{array}{l}\text { Renda familiar } \\
\left(2^{\text {a }} \text { etapa }-2001\right)\end{array}$ & $-0,07$ & $-0,03$ & $-0,14$ & 0,04 & $0,38^{* *}$ \\
\hline $\begin{array}{l}\text { Número de familiares que residiam } \\
\text { com a criança }\left(2^{\mathrm{a}} \text { etapa - 2001) }\right.\end{array}$ & $-0,42^{* *}$ & $-0,45^{* *}$ & $-0,47^{* *}$ & $-0,28$ & 0,13 \\
\hline $\begin{array}{l}\text { Número de familiares que residiam } \\
\text { com a criança }\left(3^{\mathrm{a}} \text { etapa }-2005\right)\end{array}$ & $-0,35^{*}$ & $-0,35^{* *}$ & $-0,37^{* *}$ & $-0,22$ & $-0,11$ \\
\hline
\end{tabular}

Legenda: $*=p<0,05 ; * *=p<0,01$. 
lação negativa entre o escore no SRQ-20 e a renda materna, renda familiar e quantidade de horas que a mãe passava com a criança na terceira etapa $(r=0,32 ; p<0,05)$. A renda familiar apresentou correlação negativa com o escore do BDI $(r=-0,34 ; p<0,05)$, e com o escore no SRQ-20 $(r=$ $-0,28 ; p<0,05)$. Por fim, a renda materna correlacionou-se negativamente com a quantidade de horas que ela passava com a criança.

\section{Discussão}

Em relação aos dados socioeconômicos e familiares descritos, as principais características da amostra são o baixo nível socioeconômico e a situação de vulnerabilidade das crianças, que as expõem a condições desfavoráveis para seu desenvolvimento. Conforme citado anteriormente, muitas crianças desse estudo $(70,7 \%)$ foram consideradas como sendo de "alto risco" pela medida "Indicadores de Risco para o Desenvolvimento Infantil”, na segunda etapa da pesquisa. Segundo a Organização Mundial de Saúde (World Health Organization - WHO, 2009), a sobrevivência e o desenvolvimento da criança dependem de condições (ou do atendimento) de necessidades básicas para a vida; entre elas, um ambiente saudável, seguro e limpo é fundamental. Se for considerado o que aponta o Departamento Intersindical de Estatística e Estudos Econômicos - DIEESE (2005), pode-se perceber que a renda média das famílias, naquela época (terceira etapa do estudo) era aproximadamente a metade do valor esperado. Em 2005, o salário mínimo necessário $^{3}$, segundo o DIEESE, era de $\mathrm{R} \$ 1.500,00$ e as famílias estudadas apresentavam renda média de $\mathrm{R} \$ 684,00$. Sendo assim, essas famílias encontravam-se distantes de uma condição considerada ideal. De fato, as famílias estudadas eram pertencentes à categoria "baixo inferior" na medida de nível de pobreza e pertenciam, em sua maioria, à classe C (46,3\%) e D (35,2\%). Considerando essas classificações, as crianças da amostra estudada estavam expostas a condições de risco para a sua saúde, o que poderia impactar no seu desenvolvimento e desempenho cognitivo (inclusive na leitura). Entretanto, a média geral de desempenho das crianças nas tarefas de leitura (de palavras e de texto) foi alta, considerando que a amostra variou entre $2^{\mathrm{a}}$ e $5^{\mathrm{a}}$ série. Nesse caso, outros fatores relacionados mais diretamente à criança (por exemplo, competência social, auto-estima, inteligência, capacidade para resolver problemas), assim como as experiências compensadoras proporcionadas, por exemplo, por um ambiente escolar favorável (Cecconello \& Koller, 2000; Papalia, Olds, \& Feldman, 2006), podem ter funcionado como fatores protetores. Ainda, o acompanhamento dessas famílias pelos serviços de saúde e pela

3 Corresponde ao "salário mínimo fixado em lei, nacionalmente unificado, capaz de atender às suas necessidades vitais básicas e às de sua família, como moradia, alimentação, educação, saúde, lazer, vestuário, higiene, transporte e previdência social, reajustado periodicamente, de modo a preservar o poder aquisitivo, vedada sua vinculação para qualquer fim" (Constituição da República Federativa do Brasil, capítulo II, Dos Direitos Sociais, artigo $7^{\circ}$, inciso IV). A família considerada é de dois adultos e duas crianças. Ponderando-se o gasto familiar, chega-se ao salário mínimo necessário. própria equipe de pesquisadores do estudo de coorte pode ter atenuado os riscos para o desenvolvimento das crianças, servindo como fator de proteção.

Ainda no que tange aos fatores de proteção, sabe-se que a interação com os pais, de maneira geral, e, mais particularmente, a estimulação, o reforçamento positivo e o engajamento desses nas atividades escolares dos filhos, são fatores importantes relacionados ao desempenho escolar, discutidos por diversos autores (Costa, Cia, \& Barham, 2008; D’Avila-Bacarji et al., 2005; Ferreira \& Marturano, 2002; Marturano, 2006; Okano et al., 2004). O relacionamento pais-criança dá suporte ao aprendizado da criança e fornece subsídios para que estas obtenham sucesso acadêmico e motivação para aprender (Carlton \& Winsler, 1999). Entretanto, os resultados mostraram que, quanto maior o número de pessoas que habitavam a mesma residência, mais as mães apresentavam sintomas depressivos ou outros problemas emocionais (observados a partir do escore do BDI na primeira e segunda etapas e no escore do SRQ-20 na segunda etapa). Também se observou que a mãe com problemas emocionais (conforme o escore no SRQ-20) passava menos tempo com seu filho (terceira etapa do estudo) Assim, ambas as condições (saúde mental da mãe e maior número de pessoas que residem com a criança) impactaram no desempenho em leitura de palavras, no caso deste estudo. Ainda, as consequências que os problemas emocionais provocam também o fazem.

A situação emocional da mãe dificulta a sua interação com a criança (Radke-Yarrow \& Zahn-Waxler, 1990), pois, quando deprimidas, as mães tendem a ser mais irritadas, ansiosas, tristes, menos comunicativas, mais propensas a punir e menos afetuosas (Mendes, Loureiro, \& Crippa, 2008; Pinto, Padovani, \& Linhares, 2009). Dessa forma, os comportamentos de apoio (como elogios e cuidados), tão importantes para incentivar a criança em relação à aprendizagem, tendem a ser menos frequentes (Radke-Yarrow \& Zahn-Waxler, 1990). Situações de leitura de histórias para a criança, por exemplo, apontadas como importantes para o desenvolvimento da leitura (Duursma et al., 2008; Evans, Shaw, \& Bell, 2000; Foy \& Mann, 2003; Haney \& Hill, 2004; Kuo et al., 2004; Sénéchal \& LeFreve, 2002), exigem disponibilidade emocional e de tempo da mãe e, por isso, tendem a ser menos freqüentes quando estas se encontram deprimidas.

Em relação à associação do desempenho em leitura e a psicopatologia materna ter se mostrado significativa apenas na primeira etapa de coleta (quatro meses do bebê), destaca-se que alguns estudos apontam resultados similares. Autores como Kurstjens e Wolke (2001) encontraram relação negativa entre desempenho acadêmico e em testes de QI e depressão materna apenas quando o episódio depressivo ocorria aproximadamente nos primeiros três meses após o nascimento da criança.

Somado a isso, além da relação direta com a situação emocional da mãe, quanto mais pessoas residem no mesmo ambiente, menos tempo a mãe consegue se dedicar à criança, como foi observado pela correlação entre essas variáveis $(r=-0,33 ; p<0,05)$. Como mencionado, atividades como ler para as crianças demandam tempo e concentração, tanto dos pais quanto dos filhos. Desse modo, os resultados apontam que, se existem muitas pessoas coabitando, as mães tendem a ter menos tempo para proporcionar leitura 
no ambiente doméstico. A criança, por sua vez, não adquire experiência em ler. Especialmente a leitura precisa das palavras irregulares, que são aquelas que possuem relação grafofonêmicas ambíguas (Salles \& Parente, 2002a), ou seja, aquelas nas quais a correspondência entre a grafia e o som das letras não é regular, depende da prática de leitura (Stanovich, Siegel, \& Gottardo, 1997), sendo geralmente aprendidas depois das palavras regulares no currículo escolar (Salles \& Parente, 2008). Assim, é preciso ler várias vezes a palavra, até que a criança seja capaz de reconhecê-la automaticamente quando a lê.

Nessa perspectiva, para investigar o quanto a experiência pode influenciar o desempenho em leitura, mais especificamente, avaliar a consciência fonológica, Foy e Mann (2003) examinaram crianças de quatro a seis anos. Os resultados mostraram uma associação direta entre a exposição de leitura em casa e a consciência fonológica, um processo cognitivo básico para a leitura (Fletcher et al., 2007; Salles \& Parente, 2006, 2008; Sternberg \& Grigorenko, 2003), bem como uma associação indireta entre o conhecimento de letras e vocabulário, e a exposição de leitura no ambiente doméstico. Sabe-se que, nas famílias em que a experiência com as palavras escritas não pode ser proporcionada, as crianças podem apresentar déficits na consciência fonêmica em relação àquelas de melhores condições financeiras e sociais (Foy \& Mann, 2003; Peeters et al., 2009). Um outro estudo, realizado por Sénéchal e LeFreve (2002) com 168 crianças, integrantes de um estudo longitudinal de cinco anos, mostrou que a exposição prévia à leitura no ambiente doméstico estava relacionada ao desenvolvimento do reconhecimento de palavras na $1^{\text {a }}$ série escolar e à compreensão de leitura, na $3^{\mathrm{a}}$ série.

No presente estudo, foi observado, também, que, quanto mais pessoas habitavam a mesma casa, maior a dificuldade de ler palavras regulares (aquelas em que as letras sempre representam o mesmo som, ou as letras estão no seu contexto mais comum), que geralmente são as mais fáceis de serem lidas, pois independem da estratégia (fonológica ou lexical) que será utilizada (Salles \& Parente, 2008). Além disso, essas são as primeiras palavras a serem ensinadas durante o processo de alfabetização. Se a criança não lê as palavras regulares, provavelmente não possui a habilidade básica para ler, que implica o reconhecimento da relação entre as letras e os sons (Sternberg \& Grigorenko, 2003). Esse achado também reforça a hipótese levantada anteriormente de que, nos lares em que as famílias são mais numerosas, talvez pelo maior número de filhos, as mães não se encontrem tão disponíveis para realizar com a criança atividades que envolvem a leitura. Por sua vez, a relação positiva encontrada entre a compreensão em leitura textual e a renda familiar possivelmente se deve ao fato de que as famílias com maior renda possuem mais condições de fornecer os materiais anteriormente citados - livros, brinquedos, jogos - e maiores possibilidades de atividades de lazer, apontados como importantes recursos para o desempenho escolar, incluindo a leitura (Andrada et al., 2008; Foy \& Mann, 2003; Peeters et al., 2009; Skibbe et al., 2008; Smith \& Strick, 2001). O desenvolvimento da compreensão textual é consequência do domínio do código escrito, da automatização dos processos lexicais e sua integração no contexto de leituras de palavras ou de texto, construção de inferências, motivação, monitoramento e habilidades metacognitivas (Sternberg \& Grigorenko, 2003).

Além disso, os resultados deste estudo apontaram que a renda correlacionou-se com o escore do BDI, com o escore do SRQ-20 e com o número de pessoas que habitavam a mesma casa que a criança. Esses resultados sugerem que as mães tendem a ter menos problemas emocionais quando possuem melhor nível socioeconômico e, assim, podem se envolver em atividades (como as escolares) com seus filhos, especialmente quando as famílias são menores. Consoante a esses resultados, Andrada et al. (2008) realizaram um estudo com crianças catarinenses de cinco e seis anos de idade, de centros públicos de educação infantil, encontrando que as crianças com melhor desempenho escolar eram aquelas cujas mães tinham maior grau de escolaridade e cujas famílias recebiam maiores salários. Da mesma forma, em um estudo com uma amostra de 100 crianças com dificuldades na aprendizagem escolar, Marturano (1999) observou que aquelas cujas famílias eram de menor nível socioeconômico apresentaram dificuldades até mesmo para obter meios de suprir dificuldades neurocognitivas, devido à falta de recursos estimulantes para o seu desenvolvimento.

No presente estudo, verificou-se também correlação negativa entre o número de pessoas que moravam na mesma casa que a criança e a escolaridade da mãe $(r=-0,31 ; p<$ $0,05)$. Observa-se que, além de não poderem dedicar muito tempo à criança, as mães desse estudo têm baixo nível de escolaridade e talvez não possuam condições suficientes para auxiliar nas tarefas escolares dos filhos. De fato, Haney e Hill (2004) indicaram que pais com baixo nível de escolaridade têm mais dificuldade para auxiliar as crianças na realização das tarefas escolares, influenciando de alguma forma o seu desempenho, já que a criança precisa de auxílio de uma outra pessoa mais competente para dominar uma tarefa.

Em resumo, nessa amostra, a variável número de familiares que residem com a criança explicou, sozinha, grande parte do desempenho em leitura de palavras. Entende-se que essa variável impacta no desempenho escolar e em leitura da criança porque a ela associam-se menos tempo para os pais disponibilizarem atenção e cuidados à criança e participarem de atividades educativas, além de uma menor condição socioeconômica. Desse modo, há que se considerar que essa variável está relacionada a muitos outros aspectos psicossociais.

Tendo em vista os objetivos do presente estudo, observou-se que, de maneira sucinta, o desempenho em leitura parece estar relacionado à renda familiar, à psicopatologia da mãe na primeira infância e à quantidade de pessoas que residem com a criança na amostra estudada. Entretanto, é necessário ressaltar que algumas variáveis foram examinadas em apenas algumas etapas da pesquisa, visto que, ao longo do tempo, foram feitas adaptações no projeto inicial e algumas variáveis (e instrumentos) foram sendo acrescentadas, suprimidas ou alteradas nas coletas. Por exemplo, os sintomas depressivos da mãe foram avaliados com dois instrumentos diferentes em etapas diferentes. Desse modo, algumas relações necessitariam ser melhor investigadas em estudos posteriores, nos quais as mesmas medidas sejam empregadas em diferentes momentos do tempo. 
De qualquer modo, este trabalho, ao buscar compreender as características da população estudada, que vem sendo acompanhada pelos integrantes do referido projeto, reveste-se de importância, na medida em que, ao conhecer as características dessa amostra específica, aproxima-se de um dos objetivos do projeto: conhecer as relações entre a saúde mental dos pais, relacionamento conjugal e familiar, apoio social e o desenvolvimento infantil e da família ao longo do tempo. Uma vez conhecidas as características dessa população, podem ser pensados serviços a serem oferecidos nas instituições de saúde que atendem a região em que essas famílias residem ou mesmo em outros ambientes, para melhorar a qualidade de vida dessas pessoas. Por fim, é importante lembrar que este estudo avaliou parcialmente apenas o aspecto socioeconômico e familiar relacionado ao desempenho em leitura. Entretanto, reforça a noção de que o desempenho em leitura é resultado da interação entre diversas dimensões: biológica, ambiental, cognitiva e psicossocial, que deveriam, portanto, ser foco de investigação em estudos futuros.

\section{Referências}

Aaron, P. G., Joshi, R. M., Gooden, R., \& Bentum, K. E. (2008). Diagnosis and treatment of reading disabilities based on the component model of reading. Journal of Learning Disabilities, 41(1), 67-84.

American Psychiatric Association (APA). (2002). DSM-IV-TR: Diagnostic and Statistical Manual of Mental Disorders (4 ed.). Washington DC: Autor.

Andrada, E. G. C., Benetti, I. C., Carvalho, G. B., \& Rezena, B. S. (2008). Fatores de risco e proteção para a prontidão escolar. Psicologia: Ciência e Profissão, 28(3), 536-547.

Beck, A. T., Guth, D., Steer, R. A., \& Ball, R. (1997). Screening for major depression disorders in medical inpatients with the Beck Depression Inventory for Primary Care. Behavior Research and Therapy, 35, 785-791.

Beck, A. T., \& Steer, R. A. (1993). Beck Depression Inventory: Manual. San Antonio: Psychology Corporation.

Beck, A. T., Ward, C. H., Mendelson, M., Mock, J., \& Erbaugh, J. (1961). An inventory for measuring depression. Archives of General Psychiatry, 4, 561-571.

Carlton, M. P., \& Winsler, A. (1999). School readiness: The need for a paradigm shift. School Psychology Review, 28(3), $338-352$.

Cecconello, A. M., \& Koller, S. H. (2000). Competência social e empatia: um estudo sobre resiliência com crianças em situação de pobreza. Estudos de Psicologia (Natal), 5(1), 71-93.

Costa, C. S. L., Cia, F., \& Barham, E. J. (2008). Envolvimento materno e desempenho acadêmico: comparando crianças residindo com a mãe e com ambos os pais. Psicologia Escolar e Educacional, 11, 339-351.

Cunha, J. (2001). Manual em português das Escalas Beck. São Paulo: Casa do Psicólogo.

D’Avila-Bacarji, K. M. G., Marturano, E. M., \& Elias, L. C. S. (2005). Suporte parental: um estudo sobre crianças com queixas escolares. Psicologia em Estudo, 10(1), 107-115.
Departamento Intersindical de Estatística e Estudos Socioeconômicos - DIEESE. (2005). Anuário dos trabalhadores ( $6^{\mathrm{a}}$ ed). Retirado de http://www.dieese.org. $\mathrm{br} / \mathrm{rel} / \mathrm{rac} / \mathrm{salminMenu09-05.xml.}$

Duursma, E., Pan, B. A., \& Raikes, H. (2008). Predictors and outcomes of low-income fathers reading with their toddlers. Early Childhood Research Quarterly, 23, 351-365.

Evans, M. A., Shaw, D., \& Bell, M. (2000). Home literacy activities and their influence on early literacy skills. Canadian Journal of Experimental Psychology, 54, 65-75.

Falceto, O. G. (2002). A influência de fatores psicossociais na interrupção precoce do aleitamento materno. Tese de Doutorado, Universidade Federal do Rio Grande do Sul UFRGS, Porto Alegre, RS.

Falceto, O. G., Fernandes, C. L., Kapczinski, F., Salles, J. F., Fonseca, R. P., Grassi-Oliveira, R., ... Giugliani, E. J. (2007). Fatores associados com o desenvolvimento infantil e familiar: estudo longitudinal de uma amostra populacional de crianças em idade escolar. Projeto de pesquisa, Universidade Federal do Rio Grande do Sul, Porto Alegre, RS.

Farjado, A. P., Falceto, O. G., Berger, C. B., Ramos, D. D., Cunha, J., Livi, K. L., ... Blanck, D. (2004). A atenção à saúde da criança de zero a cinco anos de idade: protocolo. Porto Alegre: Secretaria Municipal da Saúde.

Ferreira, M. C. T., \& Marturano, E. M. (2002). Ambiente familiar e os problemas de comportamento apresentados por crianças com baixo desempenho escolar. Psicologia: Reflexão e Crítica, 15, 35-44.

Fletcher, J. M., Lyons, G. R., \& Barnes, M. A. (2007). Transtornos de aprendizagem: da identificação à intervenção. Porto Alegre: Artmed.

Foy, J. G., \& Mann, V. (2003). Home literacy environment and phonological awareness in preschool children: Differential effects for rhyme and phoneme awareness. Applied Psycholinguistics, 24, 59-88.

Frankenburg, W. K., Dodds, J., Archer, P., Shapiro, H., \& Bresnick, B. (1992). The Denver II: A major revision and restandardization of the Denver Developmental Screening Test. Pediatrics, 90(3), 477-479.

Haney, M., \& Hill, J. (2004). Relationship between parent-teaching activities and emergent literacy in preschool children. Early Child Development and Care, 174, 215-228.

Hutz, C. S., Koller, S. H., \& Bandeira, D. R. (1996). Resiliência e vulnerabilidade em crianças em situação de risco. Coletâneas da ANPEPP, 1(12), 79-86.

Joshi, R. M., \& Aaron, P. G. (2000). The component model of reading: Simple view of reading made a little more complex. Reading Psychology, 21, 85-97.

Kim, Y. S. (2009). The relationship between home literacy practices and developmental trajectories of emergent literacy and conventional literacy skills for Korean children. Reading and Writing, 22(1), 57-84.

Kuo, A. A., Franke, T. M., Regalado, M., \& Halfon, M. (2004). Parent report of reading to young children. Pediatrics, 113(6), 1944-1951.

Kurstjens, S., \& Wolke, D. (2001). Effects of maternal depression on cognitive development of children over the first 7 years of life. Journal of Child Psychology and Psichiatry, 42(5), 623-636. 
Mari, J. J., \& Williams, P. (1986). A validity study of a psychiatric screening questionnaire (SRQ-20) in primary care in the city of Sao Paulo. The British Journal of Psychiatry, 148, 23-26.

Marturano, E. M. (1999). Recursos no Ambiente Familiar e Dificuldades de Aprendizagem na Escola. Psicologia: Teoria e Pesquisa, 15, 135-142.

Marturano, E. M. (2000). Ambiente familiar e aprendizagem escolar. In C. A. R. Funayama (Ed.), Problemas de aprendizagem: enfoque muldisciplinar. Campinas: Alínea.

Marturano, E. M. (2006). O inventário de Recursos do Ambiente Familiar. Psicologia: Reflexão e Crítica, 19(3), 498-506.

Marturano, E. M., Alves. M. V., \& Santa Maria, M. R. (1996). Crianças com dificuldades escolares: Recursos e adversidades na família [Resumo]. In Sociedade Brasileira de Psicologia (Ed.), Resumos de comunicações cientificas, XXVI Reunião Anual de Psicologia (p.117). Ribeirão Preto: SP.

Marturano, E. M., \& Ferreira, M. C. T. (2004). A criança com queixas escolares e sua família. In E. M. Marturano, M. B. M. Linhares \& S. R. Loureiro (Eds.), Vulnerabilidade e Proteção (pp. 217-2502). São Paulo: Casa do Psicólogo.

Mendes, A. V., Loureiro, S. R., \& Crippa, J. A. S. (2008). Depressão materna e a saúde mental de escolares. Revista de Psiquiatria Clínica, 35(5), 178-186.

Noble, K. G., Wolmetz, M. E., Ochs, L. G., Farah, M. J., \& McCandliss, B. D. (2006). Brain-behavior relationships in reading acquisition are modulated by socioeconomic factors. Developmental Science, 9(6), 642-654.

Okano, C. B., Loureiro, S. R., Linhares, M. B. M., \& Marturano, E. M. (2004). Crianças com dificuldades escolares atendidas em programa de suporte psicopedagógico na escola: avaliação de autoconceito. Psicologia: Reflexão e Crítica, 17(1), 121-128.

Oliveira, E. P. M., Guerreiro, M. M., Guimarães, C. A., BrandãoAlmeida, I. L., Montenegro, M. A., Cendes, F., \& Hage, S. R. V. (2005). Caracterização das manifestações linguísticas de uma família com Síndrome Perisylviana. Pró-Fono Revista de Atualização Científica, 17(3), 393-402.

Papalia, D., Olds, S., \& Feldman, R. (2006). Desenvolvimento humano. Porto Alegre: Artmed.

Peeters, M., Verhoeven, L., Moor, J., Balkom, H., \& Leeuwe, J. (2009). Home literacy predictors of early reading development in children with cerebral palsy. Research in Developmental Disabilities, 30, 445-461.

Pinto, I. D., Padovani, F. H. P., \& Linhares, M. B. M. (2009). Ansiedade e depressão materna e relatos sobre o bebê prematuro. Psicologia: Teoria e Pesquisa, 25(1), 75-83.

Radke-Yarrow, M., \& Zahn-Waxler, C. (1990) Research on affectively ill parents: Some considerations for theory and research on normal development. Development and Psychopathology, 2, 349-366.

Salles, J. F. (2005). Habilidades e dificuldades de leitura e escrita em crianças de $2^{a}$ série: abordagem neuropsicológica cognitiva. Tese de Doutorado, Universidade Federal do Rio Grande do Sul, Porto Alegre, RS.

Salles, J. F., \& Parente, M. A. P. P. (2002a). Processos cognitivos na leitura de palavras em crianças: Relações com compreensão e tempo de leitura. Psicologia: Reflexão e Crítica, 15(2), 321-331.
Salles, J. F., \& Parente, M. A. M. P. (2002b). Relação entre os processos cognitivos envolvidos na leitura de palavras e as habilidades de consciência fonológica em escolares. Pró-Fono Revista de Atualização Científica, 14(2), 141-286.

Salles, J. F., \& Parente, M. A. M. P. (2004). Compreensão textual em alunos de segunda e terceira séries: uma abordagem cognitiva. Estudos de Psicologia, 9(1), 71-781.

Salles, J. F., \& Parente, M. A. M. P. (2006). Heterogeneidade nas estratégias de leitura/ escrita em crianças com dificuldades de leitura e escrita. Psico, 37(1), 83-90.

Salles, J. F., \& Parente, M. A. M. P. (2007). Relação entre o desempenho infantil em linguagem escrita e percepção do professor. Cadernos de Pesquisa, 37(132), 687-709.

Salles, J. F., \& Parente, M. A. M. P. (2008). Variabilidade no desempenho em tarefas neuropsicológicas entre crianças de $2^{\mathrm{a}}$ série com dificuldades de leitura e escrita. Arquivos Brasileiros de Psicologia, 60, 1-10.

Salles, J. F., \& Parente, M. A.M. P. (2009). As dificuldades de leitura e escrita em crianças/adolescentes: abordagem neuropsicológica cognitiva. In V. G. Haase, F. O. Ferreira \& F. J. Penna (Eds.), Aspectos biopsicossociais da saúde na infância e adolescência (pp. 353-373). Belo Horizonte: COOPMED.

Santos, R. S., Araújo, A. P. Q. C., \& Porto, M. A. S. (2008). Early diagnosis of abnormal development of preterm newborns: assessment instruments. Journal of Pediatric, 84(4), 288-299.

Sénéchal, M., \& LeFreve, J. A. (2002). Parental involvement in the development of children's reading skill: A five-year longitudinal study. Child Development, 73(2), 445-460.

Skibbe, L. E., Justice, L. M., Zucker, T. A., \& McGinty, A. S. (2008). Relations among maternal literacy beliefs, home literacy practices, and the early literacy skills of preschoolers with specific language impairment. Early Education and Development, 19, 68-88.

Smith, C., \& Strick, L. (2001). Dificuldades de aprendizagem de A a Z. Porto Alegre: Artmed.

Snowling, M. J., Muter, V., \& Carroll, J. (2007). Children at family risk of dyslexia: a follow-up in early adolescence. Journal of Child Psychology and Psychiatry, 48(6), 609-618.

Stanley, C., Murray, L., \& Stein, A. (2004). The effect of postnatal depression on mother-infant interaction, infant response to the Still-face perturbation, and performance on an Instrumental Learning task. Development and Psychopathology, 16(1), 1-18.

Stanovich, K. E., Siegel, L. S., \& Gottardo, A. (1997). Converging evidence for phonological and surface subtypes of reading disability. Journal of Educational Psychology, 89, 114-127.

Sternberg, R. J., \& Grigorenko, E. L. (2003) Crianças Rotuladas. São Paulo: Artmed.

The Psychological Corporation. (1999). Wechsler Abbreviated Scale of Intelligence Manual. San Antonio: Autor.

Yates, D. B., Trentini, C. M., Tosi, S. D., Corrêa, S. K., Poggere, L. C., \& Valli, F. (2006). Apresentação da Escala de Inteligência Wechsler Abreviada (WASI). Avaliação Psicológica, 5(2), 227-233.

WHO (World Health Organization). (1994). A user's guide to the self reporting questionnaire (SRQ). Geneva: Division of Mental Health, World Health Organization. 
WHO (World Health Organization). (2009). Third international conference on children's health and the environment: from knowledge and research to policy and action. International Conference on Children's Health and the Environment. Geneva: Division of Mental Health, World Health Organization.

Winter, L. B., Steer, R. A., Jones-Hicks, L., \& Beck, A. T. (1999). Screening for major depression disorders in adolescent medical outpatients with the Beck Depression Inventory for Primary Care. Journal of Adolescence Health, 24(6), 389-94.

Zimmerman, M. A., \& Arunkumar, R. (1994). Resiliency research: implications for schools and policy. Social Policy Report, 8(1), 1-18.

Recebido em 15.06.2010 Primeira decisão editorial em 30.06.2011 Versão final em 22.06.2012 Aceito em 22.06.2012

\section{Encontro Abordando o Sono: sono e processos cognitivos}

Natal, BR

28 a 29 de Novembro de 2012 